\title{
Group Decision Support Using Toulmin Argument Structures
}

\author{
CONF-9610218--1 \\ Terry Janssen* and Andrew P. Sage \\ School of Information Technology and Engineering \\ George Mason University \\ Fairfax, VA 22030
}

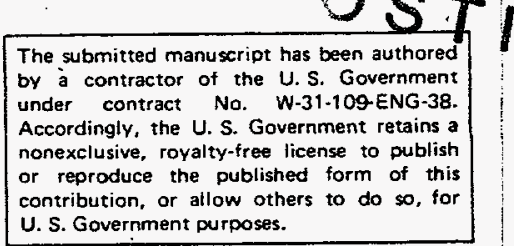

\section{ABSTRACT}

This paper addresses the need for sound science, technology, and management assessment relative to environmental policy decision making through an approach that involves a logical structure for evidence, a framed decision-making process, and an environment that encourages group participation. Toulmin-based logic possesses these characteristics and is used as the basis for development of a group decision support system. This system can support several user groups, such as pesticide policy-making experts, who can use the support system to state arguments for or against an important policy issue, and pest management experts, who can use the system to assist in identifying and evaluating alternatives for controlling pests on agricultural commodities. The resulting decision support system assists in improving the clarity of the lines of reasoning used in specific situations; the warrants, grounds, and backings that are used to support claims and specific lines of reasoning; and the contradictions, rebuttals, and arguments surrounding each step in the reasoning process associated with evaluating a claim or counterclaim. Experts and decision makers with differing views can better understand each other's thought processes. The net effect is enhanced communications and understanding of the whole picture and, in many cases, consensus on decisions to be taken.

\section{INTRODUCTION}

Policy decision making is a process of identifying and selecting policy options. Some policy options can be understood and properly considered only by tapping the expertise of experts in relevant scientific disciplines. This process, called science assessment, involves collecting, understanding, and properly considering science to determine the viability (outcome) of policy options. It is performed for answering policy questions, to the extent possible, on the basis of "sound, objective, unbiased" science.

It is often difficult for policy makers to understand how content specialty experts, scientists and engineers, arrive at their judgments and what to believe when two experts give contradictory answers. There is a plethora of research, often reaching different conclusions, and it is organized and presented in a way that is difficult for a policy maker to understand in relation to the policy issue that needs resolution. Also, the science or technology assessment and review process often takes too long because experts are inefficiently networked. The problem is further compounded by lack of an efficient structure for eliciting complete, precise, and accurate claims, rebuttals, and counterclaims directly from the experts, in a way that is practical and efficient. After the assessment, the details of how and why a science assessment

* Dr. Janssen is also with the Argonne National Laboratory, and is working under contract to the U.S. Department of Agriculture, a major sponsor of this work. question was answered are often lost because of incomplete documentation.

Figure 1 illustrates the cycle that policy makers go through in large science and technology assessments that require multiple experts to fully cover the range of issues surrounding the policy question. The problem begins with the need to assess an option, as depicted in the top left quadrant of the figure. Experts need to be tapped (top right) to provide expert opinions and supporting evidence, but the experts are often located around the country or world. The process is time-consuming because the experts need to be contacted and provided with explanations of what expertise is needed to provide useful input to the assessment. The expert's response is usually a summary, without the details of how they came to that assessment. The grounds and backing for the assessment are usually not well documented. If two experts give contradictory assessments, it may be difficult for the policy maker to understand how their arguments compare. The policy maker must choose by deciding which argument, or hypothesis, has the greatest degree of support. When information is missing, the policy maker must go back to the experts for clarifications. This process of considering the science or technology, identifying missing information, and going back to the experts can take weeks or longer. As a result, important details may be omitted.

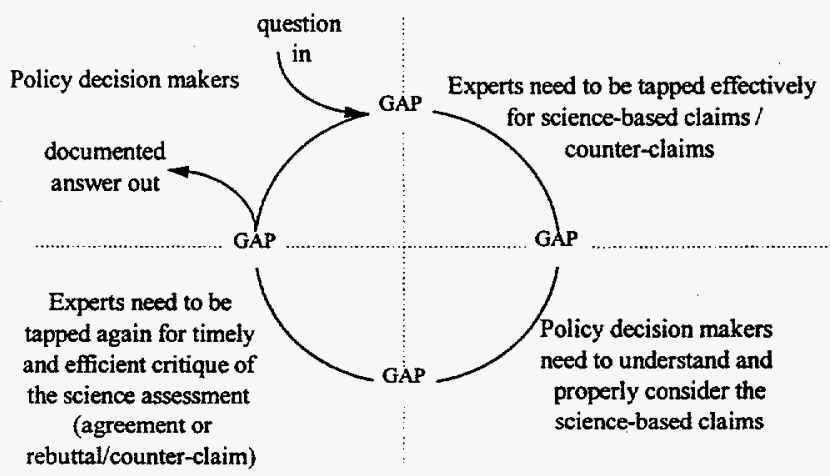

Figure 1. The Gaps between Policy Makers and Experts in Science and Technology Assessment

Policy makers often find it difficult to understand and consider an expert's science or technology assessment and how it relates to the given policy question, as indicated in the lower right portion of Figure 1, because the assessment lacks details, such as the grounds and backing upon which conclusions were drawn and the confidence that the expert has in those conclusions.

- It is difficult for policy makers to understand and assess the arguments put forth by experts who have conflicting opinions.

- It is difficult for policy makers to see hidden bias on the part of the expert. 


\section{DISCLAIMER}

This report was prepared as an account of work sponsored by an agency of the United States Government. Neither the United States Government nor any agency thereof, nor any of their employees, makes any warranty, express or implied, or assumes any legal liability or responsibility for the accuracy, completeness, or usefulness of any information, apparatus, product, or process disclosed, or represents that its use would not infringe privately owned rights. Reference herein to any specific commercial product, process, or service by trade name, trademark, manufacturer, or otherwise does not necessarily constitute or imply its endorsement, recommendation, or favoring by the United States Government or any agency thereof. The views and opinions of authors expressed herein do not necessarily state or reflect those of the United States Government or any agency thereof. 


\section{DISCLAMMER}

Portions of this document may be illegible in electronic image products. Images are produced from the best available original document. 
- It is difficult to see how the arguments presented by the expert relate to the original science or technology question.

Experts intuitively assess the degree of support the scientific evidence brings to bear on their claims or hypotheses, but their assessments are usually not explicitly stated and recorded. Peer review is usually required, but there may be no means whereby the peer review group can systematically review the assessment through use of all the grounds and backing that led to the assessment conclusions. The information may not be structured so that the reviewers can see the grounds and backing for the claim; therefore, it is difficult to assess the degree of support associated with the hypothesis or claim.

After policy makers collect the pieces of the assessment and bring them together in the context of the policy question, they often ask another group to provide a peer review. If an expert does not agree with a claim made in the assessment, he or she must provide a rebuttal or a counterclaim. Here again is a lack of structure and systematic means for this process. The process is time-consuming, often taking many months.

Once the expert's review is provided, the policy maker must be able to understand and properly consider the expert's claims and counterclaims. If the assessment is adequate, as judged by those conducting the assessment, then it needs to be documented for use by policy makers. If it is not adequate, the assessment process continues through another cycle. This is a time-consuming and costiy process, and efforts to make it more efficiently and timely are needed.

We propose an approach for filling these gaps between policy makers and experts, and we discuss how this approach fits within the framework of a Group Decision Support System (GDSS). First, we define some terms:

- An expert is someone considered an expert by peers because of a high degree of knowledge in a specific field, in a discipline relevant to the claim, such as chemistry, biology, entomology, or high-performance computing.

- A claim is an assertion that truth is associated with a statement.

- A hypothesis $(\mathrm{H})$ is an assumption or concession made for the sake of arguing this claim.

- The hypothesis is supported or refuted by evidence in the form of grounds (G) for the claim.

- To accept a hypothesis, the degree of support (S) for the hypothesis $(\mathrm{H})$ must be greater than that for the counterclaim or null hypothesis $(\mathrm{Hc})$.

- If the support (Sc) for the counterhypothesis (Hc) is greater, then we reject the hypothesis.

- Finally, policy decisions are strategic, and tactical decisions are more operational in nature and are used to implement a policy decision.

\section{TOULMIN LOGIC}

Toulmin [1] [2] suggests that logic theories come from the fields of psychology, sociology, technology, and mathematics. The paradoxes and pitfalls of mathematical logic are discussed in these seminal works in which he establishes an informal logic to address these paradoxes and pitfalls - a logic that is frequently referred to as Toulmin logic. It has been called revolutionary [3]. However, the philosophy of logic remains a topic of debate and research [4] [5].
We refer to Toulmin logic as Toulmin structures. These structures, suggested in Figure 2, are a graphic representation of the components of an argument, including a claim and the grounds from which the claim is inferred. Toulmin structured argument and its component parts are defined as follows:

- A claim is an assertion of a truth.

- Grounds provide the basis for the inference to that claim.

- Grounds can be categorized by the means in which are warranted:

- P Empirical observations,

- P Expert judgments,

- P Enumerative induction (statistics),

- P Experiments (hypothesis test), and

- P Direct fact.

- A warrant serves as assurance for a claim and identifies the (informal) logic and the backing used in making the claim.

- The backing describes how the claim is supported. It is a concise synopsis of the evidence and logic used in the warranting process.

- The modality is a subjective statement concerning the probability of the claim being true, given the grounds.

- A rebuttal is a statement that weakens the modality of the claim based on evidence that the claim may not be true. A rebuttal is stated explicitly as a statement on a claim.

- A counterclaim serves as a rebuttal in that it also weakens or nullifies the claim to some extent.

The grounds and backing of a claim can be any relevant quantitative information from experimental or empirical test results, statistical hypothesis testing, or results from modeling and simulation. The grounds and backing can also be any relevant qualitative information from direct facts, such as laws and regulations, observations, and the expert's professional or personal opinions.

We selected Toulmin structures for this purpose after considering several possible structures: influence diagrams and Bayesian networks, which are oriented toward quantitative analysis and are not easy to build; structured modeling and conceptual modeling, which can be used to construct a Toulmin structure; and Wigmore structured evidence, which comes the closest to Toulmin and is an argument structure based on an elaborate set of symbols. The Wigmore approach, although closest to our Toulmin-based approach, is much more complicated than Toulmin. The Toulmin structure, is easy to use and understand and is widely applicable. Our studies suggest that when the Toulmin structure is framed appropriately, it is easy for the policy maker to understand and to use in assessing science and technology assessment questions. Many of these approaches are described in the literature [6] [7]. There have also been a number of other studies concerning the implementation of Toulmin-based logic [8] [9] [10] [11].

A natural language equivalent to a Toulmin argument always exists because structure can be imposed with words rather than graphically with boxes and lines. However, there are several advantages to a graphical or structured depiction. First, visualization eases comprehension. The components of the argument are explicitly represented, so it is easier to identify the particular elements of an argument. These elements serve as place holders and thereby facilitate elicitation of these elements. The person filling in the boxes can see what is missing as well as the 


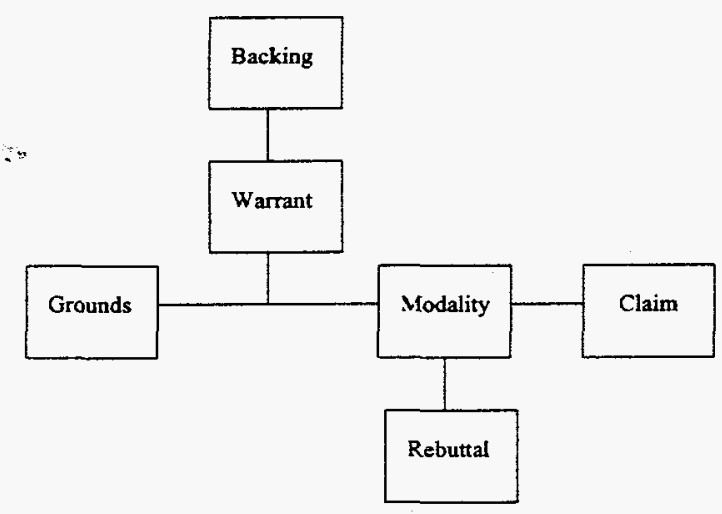

Figure 2. Toulmin Structure

reasoning that has been put forth. It is easier to compare arguments between multiple experts, and between claims $(\mathrm{H})$ and counterclaims $(\mathrm{Hc})$, than between statements in generally unstructured discourse.

Because of the explicit nature of Toulmin structures, they are generally more objective than arguments that are not made explicit. This is usually the case since it is harder to hide bias when the grounds and backing of an argument are explicit. For example, consider two experts with conflicting interests, one who is an industrialist biased against environment and another who is an environmentalist biased against industry. Each is asked to represent claims about whether use of a pesticide, say malathion, is excessively risky. Each person has to support his or her claims with appropriate grounds and warrants, and the perspective that each person takes is generally easy to see through examination of the grounds and warrants.

\section{EMPIRICAL ASSESSMENT}

To test our hypothesis that Toulmin structure bridges the gap between expert and policy maker, we conducted an empirical test [12], [13]. A test case was independently selected - a science assessment of pest management research area alternatives, part of a high-priority national program to achieve integrated pest management on $75 \%$ of U.S. crops by the year 2000 . The underlying problem is that of pest damage to agriculture, which causes a loss of many billions of dollars every year.

The U.S. Department of Agriculture is developing a pest management information and GDSS. The architecture of this system is based on use of a decision support system (DSS) with three components: a dialogue generation and management system, a database management system (DBMS), and a model-based management system [14]. The DSS is currently under development. It has been used in a limited capacity to answer science assessment questions and support decisions on which crop/pest areas to fund in a 1995 request for proposal, and is currently being used in the same process in 1996. Toulmin structures have been implemented and integrated with the DSS for test purposes.

The following is an example of a science assessment question in the context of a crop and an associated pest: "Is Endosulfan a viable alternative for addressing a particular crop/pest problem?" Figure 3 shows the framing of this question with subhypotheses. The corresponding text descriptions for the elements of the Toulmin arguments are not shown. The warrant, not shown, states that we can infer this claim if we can accept at least one of the following subhypotheses (grounds):

- Endosulfan is illegal to use.

- It is unsafe for humans or the environment.

- It is not effective in controlling the targeted pest.

- It is not economical.

- It is facing genetic resistance in the pests it is supposed to control.

The warrant is documentation of how the claimant warrants his or her claims. We have accepted the hypothesis that Endosulfan, even when used according to labeling, is unsafe, and we are using it to support the claim that Endosulfan is not a viable alternative for controlling the crop/pest in question. We warrant that Endosulfan is unsafe if we can accept at least one of the subhypotheses: that it unsafe for humans or the environment. The complete details of this example, including the assessment of degree of support, can be found in [13].

The Toulmin structure had to be extended in several minor ways to map it to science assessment in the area of pest management decision making considered here. These minor extensions include the definition of claims and counterclaims, and a hierarchical structure for graphically relating Toulmin structures. We incorporated structures into a hyperlinked environment, in a software prototype, and provided other features that make Toulmin structures practical for multiple experts and policy makers to use interactively within the test case problem domain.

The purpose was to test Toulmin structures as a means of bridging the gap between experts and policy makers. This test was conducted with actual experts and policy makers doing a science assessment on the following two questions, as part of their pest management policy (strategy) decision-making process:

- If a pesticide is regulated, what are the viable alternatives?

- What is the viability of an alternative to a regulated pesticide?

An example of the latter, framed for science assessment, is illustrated in Figure 3. The purpose of this science assessment is to support research funding allocation decisions, based on scientific evidence collected and brought to bear on these questions. The test subjects were independently selected from the experts and policy makers involved in the national program responsible for this science assessment of pest management alternatives.

The results of the assessment were positive. The results suggest that it is easy for scientists to state virtually all relevant considerations by using Toulmin structures, and that Toulmin structures bridge the gap between the experts and policy makers. 


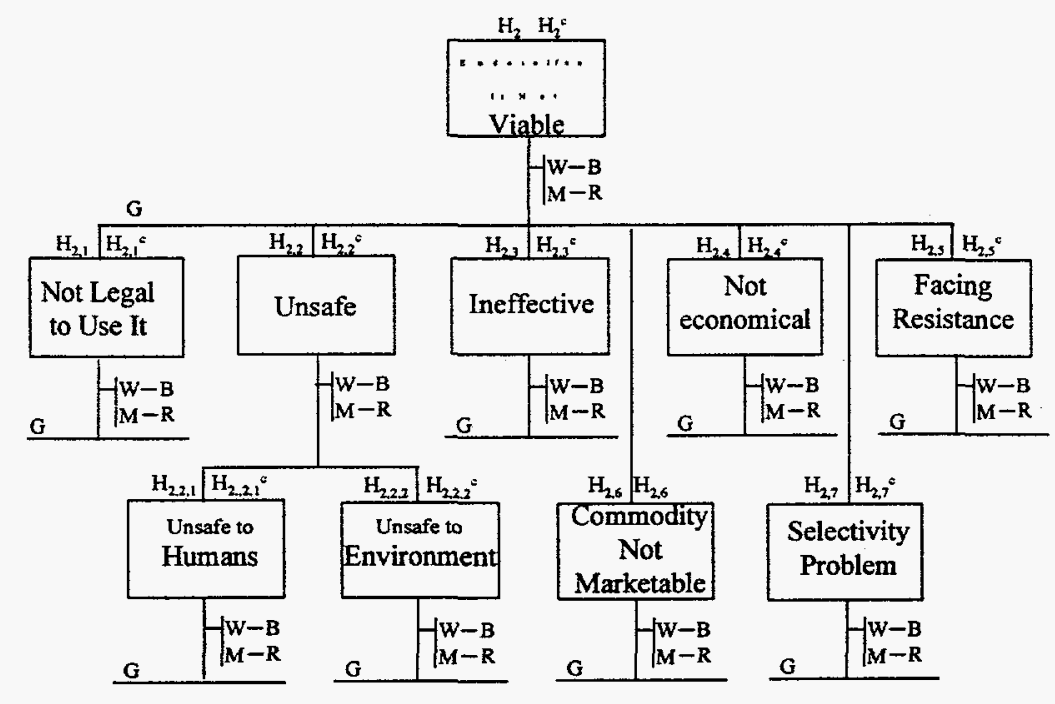

Figure 3. Example of Toulmin Argument with Hierarchical Structure

\section{GROUP DECISION SUPPORT SYSTEM FRAMEWORK}

The science assessment process described above requires group debate, assessment, and decision making. For science assessments involving several disciplines, experts need to be tapped from each discipline. In our example, these disciplines include agronomy, environmental science, economics, entomology, biology, chemistry, and plant pathology. We are exploring the use of Toulmin structure as part of a GDSS that would provide distributed access to experts and policy makers located in several locations and provide a structure for conducting scientific argument and science assessment for important policy questions.

As is now well known [14], a GDSS is a DSS that supports a group of decision makers. The group of people can be centralized at one spot or decentralized in space and/or time. Also, the decision considered by each individual in a decision-making group may or may not be the ultimate decision. The decision being considered may be sequential over time and may involve many component decisions. A GDSS provides a mechanism for group interactions. A GDSS may impose various structured processes on individuals in the group; these need to be considered in evaluating GDSS effectiveness.

Toulmin structure is a foundation for group debate and scientific argument. Experts in science or technology assessment may be in a central location in one situation and distributed in another. The Toulmin structure prototype developed for pest management policy decision making appears to fit well in both types of settings - in a peer review meeting where all experts get together and within a data communications network of distributed experts decentralized in time and space. Toulmin-based logic imposes a structure and process, consistent with the definition of a GDSS.

The science and technology assessment process is but one of many aspects of policy decision making. With Toulmin structure, the participants are not only allowed to have different interpretations but are encouraged to do so and to engage in scientific argument. The process for assessing the degree of support for hypotheses provides a means for accepting or rejecting hypotheses. Toulmin structure is a form of knowledge representation and needs to be associated with some form of human-machine dialog [15]. However, unlike most knowledge representations, Toulmin structure provides group participants with an explicit structure that can be used for a relatively complete representation of claims, and the grounds and backings that support them. The GDSS framework is favorable for gathering expert opinion. Using Toulmin structure in the GDSS also makes it appropriate for resolving science- and technologybased arguments.

Toulmin structures may also be used to document how a claim is justified. The warrant records the rules of thumb, the logic, or whatever the claimant has claimed gives license for the claim. Warrants and modalities can be categorized as domain independent and domain dependent. Toulmin $[1,2]$ indicates that the criteria or grounds for justification vary from field to field. Physiological incapacity in biology, standards for inadmissibility in jurisprudence, and impossibility in mathematical terms are different means of justifying claims. In mathematics, impossible is defined in terms of the absence or presence of a demonstrable contradiction. Impossible takes on a different meaning in social, political, economic, or scientific disciplines, such as the possibility of a pest management tactic killing beneficial insects. Criteria of possibility are all field dependent. To a large extent, entomology, chemistry, and biology are all based on the principles of science and the scientific method. These scientific disciplines have established principles upon which the claims in these disciplines need to be warranted. Domainindependent criteria for two arguments in science assessments are as follows:

- Coherence or logical consistency of the arguments;

- Congruence because the arguments must coincide;

- Cogency because the arguments must be convincing or valid; and

- The arguments must have practical utility.

We can categorize the means by which scientists or engineers might warrant an inference from grounds to claim in science assessment as follows:

- Empirical observation, when data from observation suggests that it is true;

- Expert opinion, when the source is regarded as an authority on the topic;

- Enumerative induction, when statistical significance is demonstrated;

- Results from a controlled experiment, when a test group is significantly different than a control group; or

- Results that follow from an agreed upon or imposed rule, like a law or a regulation.

Although other warrant categories are possible, it is common for experts to warrant claims in science assessment by these means. In a GDSS setting, the warrants are seen by all participants. One person might use a different warrant with the same grounds, or different grounds or the same grounds for rebuttals, counterclaims, or other variations. It would be unreasonable to expect all cases to fit neatly into these categories, but they suffice for our discussion 
here. If there is contention, or argument, the group might vote on the validity or reasonableness of the grounds and warrant, or use some other group technique to decide whether the warrant is reasonable and the claim is acceptable.

In science or technology assessment, does the warrant actually give license to"make the claim or is it just that we believe we have license? This question can be answered by analyzing the credibility of the warrant according to Landsbergen [16]. The group needs to decide whether the warrant, or the argument as a whole, is credible. However, the approach we have adopted is to crudely assess the degree of support and to accept a hypothesis if the support is greater than that for the null or counterhypothesis and to reject it if this is not the case. We propose that this assessment be done by experts within a GDSS. Consistent with the scientific method of hypothesis and test, the group could weight the evidence by using criteria agreeable to the group for the purpose of accepting or rejecting each hypothesis.

The source of the evidence is one criterion. Consider the rough categories of warrants mentioned earlier. Empirical observation gives us the right to make claims when following sound rules of inference as we can often warrant claims based on empirical observation. Inferences can generally be much stronger when based on a controlled experiment that shows significant differences between the control group and the test group. However, there are no firm rules or guidelines on what warrants are acceptable in science assessment and those that are not. For example, a controlled experiment that was not properly conducted does not necessarily give license for inference. If the experiment is improperly designed, its conclusions may be erroneous, and inference of the claim from those results would not be valid. Science assessment depends on valid warrants, and it is not always clear when a stated warrant is not valid.

In science and technology assessments, the assessment of grounds and warrants must be sound. When there is debate, Toulmin structure appears to be a practical means of science assessment. Ease of access and use by experts is critical, and implementation in a GDSS makes this possible. Experts can assess the validity or reasonableness of arguments, as well as provide supporting evidence or rebuttals and counterclaims. However, Toulmin structure has not been widely used in this capacity, and several research questions exist.

\section{COMPUTER IMPLEMENTATION ISSUES}

The test prototype was implemented in Windows 3.1 on a personal computer. It is a multi-user system - the software can be installed on several computers and the claims and counterclaims can transferred over the Internet, as was done to a limited extent for the operational test and evaluation of the prototype. To be practical in an operational setting, Toulmin structures would need to be implemented a way analogous to a GDSS. This raises many implementation questions.

Should the implementation be user mission specific at the expense of general applicability, or should it be a general-purpose implementation that is less useful to a specific group of users? Our test results suggest that mission-specific implementation, such as integration in a user-specific DSS, dramatically increases acceptance by the user group. In our test case, for example, the experts needed access to information to make claims. The capability of the test software to cut and paste that information easily into the grounds and backing field of a claim structure was rated very highly by the test subjects, because these experts depend on that information to make claims during the assessment process. We believe that the approach used for Toulmin structure implementation within the user's domain is critical and strongly influences successful use of Toulmin structures.

Our test software implementation is not like a HyperCard implementation. HyperCards provide for general-purpose statement of claims and the elements of the Toulmin structure, but they do not provide a canonic semantic as we have done to describe the claims in a machine-processable form [12,13]. The canonic semantic and a field (domain-dependent) vocabulary are key to relational database implementation. Domain-dependent mapping enables integration with a DSS, at least in the pest management test case. Further research is needed to assess the trade-offs between general-purpose and mission-specific implementations, in the contexts of different user groups working on different problems. Such research will also need to address a variety of operational issues.

\section{EVALUATION ISSUES}

The issue of how different experts will engage in scientific argument with Toulmin structure needs to be addressed. In a GDSS setting, the group can use Toulmin structure for adding claims, adding hypothesis nodes, and structuring arguments or lines of reasoning. However, further laboratory and field research is needed to identify the most appropriate operational setting for the support system. For example, should the science assessment process be controlled? Does the process work better if it is democratic or dictatorial? Should claims be limited to a small set of experts who are considered "expert" by their peers (restricted), or should anyone have the opportunity to add claims, rebuttals, and counterclaims (unrestricted)? Is it better to ask specific experts for claims on specific topics or to let the process evolve on its own in an undirected manner?

As stated earlier, GDSS has been used to elicit expert opinion but not for conducting a science assessment. Results from our empirical assessment support the notion that Toulmin structure would be an effective means of eliciting expert opinion and scientific argument in a GDSS framework. Although we have some empirical evidence from our limited test, the effectiveness of Toulmain structure remains to be demonstrated in an operational GDSS setting. Does Toulmin structure provide a more cost-effective way of conducting science assessment; are fewer meetings required; does the quality of the assessment process increase; does the quality of the assessment product increase; does time to closure decrease; is it easier to form science assessment teams with the GDSS; and does the GDSS and Toulmin structure process fit well with the group dynamics of how assessments evolve? The answer to these questions have been positive for many GDSS implementations in the past, but we need to perform laboratory and field studies to determine whether this is true when we impose Toulmin structure on the GDSS setting.

A number of issues relate to orchestration of the process itself. For example, do we need someone to oversee or facilitate the process when it involves several experts in different locations? It seems reasonable that a moderator could raise issues in the group, initiate dialogue, and bring the group to decision points on how the debate and assessment would be carried out. Should claims be reviewed by 
using the same types of techniques established for group assessment in GDSS, such as nominal group technique, Delphi, or other methods of collective inquiry? We need appropriate approaches in specific situations to answer such questions: is the warrant used to infer the claim from the grounds reasonable in context of field-dependent criteria; are "good reasons" being used to warrant the claim, and are the grounds relevant to the claim; how strongly do the grounds support the claim; and does the evidence support acceptance or rejection of the hypothesis?

The experts involved in the science assessment need to use fieldspecific criteria for warranting and assessing the degree of support, but more needs to known. Toulmin structure needs to be fielded in an operational GDSS, and additional research is needed to learn more about Toulmin structure in this environment, such as what techniques are best used for group science assessment and the fielddependent criteria that should be used for science assessment.

"Maintenance" issues also exist. When should arguments be discarded or rejected? Is it advantageous to provide archiving and retrieval on demand of all arguments, even those rejected? In most cases, successful use of a GDSS usually requires a facilitator. Do we also need a Toulmin structure database administrator to maintain the arguments? For example, adding of arguments, and no deletions, might make the argument database unwieldy. When can a claim be deleted? When does new information make the old obsolete? Should all claims be archived for potential retrieval?

Questions related to reassessment also exist. New evidence and claims may require the experts to reassess an initial group decision on whether to accept or reject hypotheses. Experience in Toulmin structures is needed in an operational GDSS before we will know how to manage reassessment within an operational GDSS.

Cost issues are also of importance. What would it cost, for example, to conduct a assessment using Toulmin structure within a GDSS setting? Our limited empirical assessment involving Toulmin structures has not provided much cost information. There is the direct cost of GDSS, whether it is a specially engineered room or distributed workstations residing on experts' desks in a remote location. There are the indirect costs, such as the expert's time. If the cost increases, does the GDSS setting provide greater quality, enough to justify the added expense? It is possible that we would find savings from reduced travel and more efficient use of the expert's time, so a distributed GDSS might actually reduce the overall costs.

Success factors relating to successful deployment of Toulmin structure are also of concern. Use of explicit Toulmin structure is an unusual way for experts to engage in argument, although our empirical evaluation suggests that it is natural to the thought process of most scientists and policy decision makers. Some users will most likely resist this new way of conducting business. However, when Toulmin structure is used within an established GDSS setting, these differences may be tolerated or even welcomed by the participants. Nevertheless, how to successfully field Toulmin structure in a GDSS is an open issue. In general, the success factors for GDSS are early success, an infrastructure to support the use of a GDSS, and a corporate champion [17]. It seems reasonable to expect that these factors would apply to Toulmin structure in the GDSS setting as well.

\section{SUMMARY}

We have proposed the use of Toulmin structure for science assessment in a GDSS framework. We reported on the development of a software prototype for testing and the results of an empirical assessment. We have not yet fielded Toulmin structures within a GDSS. However, we are exploring the use of the World Wide Web as a first attempt at providing a GDSS-like framework for conducting mission-specific science assessment.

Our current prototype provides secure desktop access for experts doing a science assessment of pest management alternatives. Experts anywhere can engage in debate and scientific argument by entering claims, rebuttals, and/or counterclaims in Toulmin structure over the Web. These arguments are brought to bear on science assessment questions and serve as an interface between the experts doing the science assessment and the policy decision makers using the assessment.

In our test prototype, we provided the capability to assess the degree of support for hypotheses that were framed as part of our test case science assessment. Our Web implementation does not currently provide the capability to assess degree of support, but we hope to have it in place soon. Our goal is to build out a limited distributed GDSS using the Web as the communications infrastructure. When this is in place, we can begin exploring the open research issues mentioned in this paper.

\section{ACKNOWLEDGMENTS}

Work performed for the U.S. Department of Agriculture through an agreement with the U.S. Department of Energy under contract number W-31-109-ENG-38.

\section{REFERENCES}

[1]Toulmin, S., The Uses of Argument, Cambridge University Press, London, 1958.

[2]Toulmin S., Rieke, R., and Janik, A., An Introduction to Reasoning, Macmillan Publishing, New York, 1984.

[3] Cooley, J., "On Mr. Toulmin's Revolution in Logic," Journal of Philosophy, Vol. LVI, No. 7, 1959, pp. 297-319.

[4]Hample, D., "The Toulmin Model and the Syllogism," Journal of the American Forensic Association, Vol. 14, Summer 1977.

[5]Brockriede, W., and Ehninger, D., "Toulmin on Argument: An Introduction and Application," Quarterly Journal of Speech, Vol. 46, 1960, pp. 44-53

[6]Sage, A. P., Systems Engineering, John Wiley and Sons, New York, 1992.

[7]Schum, D., Evidential Foundations of Probabilistic Reasoning, John Wiley and Sons, New York, 1994.

[8]Lagomasino, A., and Sage, A. P., "Representation and Interpretation of Information for Decision Support with Imperfect Knowledge," Large Scale Systems, Vol. 9, No. 2, 1985, pp. 169-181.

[9]Lagomasino, A., and Sage, A. P., "An Interactive Inquiry System," Large Scale Systems, Vol. 9, No. 3, 1985, pp. 231244.

[10]Sage, A. P., "On the Processing of Imperfect Information Using Structured Frameworks," Chapter 7 in Kandel, A. (Ed.), Fuzzy Expert Systems, CRC Press, New York, 1991, pp. 99-112. 
[11]Laskey, K. B., Chen, M. S., and Martin, A. W., "Representing and Eliciting Knowledge about Uncertain Evidence and its Implications," IEEE Transactions on Systems, Man, dnd Cybernetics, Vol. 19, No. 3, May 1989, pp. 536-545.

[12]Janssen, T., "Toulmin Based Logic in Policy Decision Making," it Miramond, M., Gauffre, P., Beheshti, R., and Zreik, K. (Eds.), A Critical Review of the Application of Advanced Technologies in Architecture, Civil and Urban Engineering, Europia Productions, Paris, 1995, pp. 315-332.

[13]Janssen, T., Toulmin Argument Structures for Science Assessment, Doctoral Dissertation, George Mason University, Fairfax, VA, 1996.

[14]Sage A., Decision Support Systems Engineering, John Wiley and Sons, New York, 1990.

[15]Sage, A. P., and Lagomasino, L., "Knowledge Representation and Man-Machine Dialog," in Rouse, W. B. (Ed.), Advances in Man Machine Systems Research, Vol. 1, JAI Press, Greenwich CT, 1984, pp. 223-260.

[16]Landsbergen, D., A Credibility Model of Policy Decision Making: Effects of Computer Media, Advocacy, and Policy Arguments, Ph.D. Dissertation, Syracuse University, 1987.

[17]Rockart, J., "Critical Success Factors," Sloan Management Review, March-April, 1979. 